\title{
A FIXED POINT THEOREM-FREE APPROACH TO WEAK ALMOST PERIODICITY
}

\author{
BY
}

\author{
WILLIAM A. $\operatorname{VEECH}\left({ }^{1}\right)$
}

\begin{abstract}
In this paper we present a generalization of the Eberlein, de Leeuw and Glicksberg decomposition theorem for weakly almost periodic functions which does not rely on any fixed point theorem for its proof. A generalization of the Ryll-Nardzewski fixed point theorem is given.
\end{abstract}

1. Introduction. In the present note we generalize certain results pertaining to the theory of weakly almost periodic functions, such as the Eberlein decomposition theorem [4] (in the general form given to it by de Leeuw and Glicksberg [2]) and the Ryll-Nardzewski fixed point the orem [18]. One feature of our approach is that the decomposition theorem is obtained without recourse to the Ryll-Nardzewski (or any other) fixed point theorem. We begin with some notations and standard definitions.

A flow $(\Sigma, X)$ consists of a semigroup $\Sigma$, a topological space $X$, and a homomorphism from $\Sigma$ to the semigroup of continuous maps from $X$ to $X$ (in which composition of maps is the binary operation). As is customary, we suppress the homomorphism and write simply $\sigma x$ for the image of $x \in X$ under (the image of) $\sigma \in \Sigma$. If $\mathcal{U}$ is a uniformity compatible with the topology of $X$, we can speak of $(\Sigma, X)$ being distal relative to $\mathcal{U}$. This means for every pair $x, x^{\prime} \in X, x \neq x^{\prime}$, there exists $U \in \mathcal{U}$ such that $\left(\sigma x, \sigma x^{\prime}\right) \notin U, \sigma \in \Sigma$. If the uniformity is understood or unique (as in the case of $X$ compact Hausdorff) we say simply that $(\Sigma, X)$ is distal. If $\mathcal{U}$ is defined by a metric $D(\cdot, \cdot)$, then distality relative to $\mathcal{U}$ is equivalent to $\inf _{\sigma \in \Sigma} D\left(\sigma x, \sigma x^{\prime}\right)>0$ for $x \neq x^{\prime}$.

If $(\Sigma, X)$ is distal with $X$ compact Hausdorff, there is an associated object $E=E(\Sigma, X)$, called the Ellis group. To construct $E$, let $\Sigma$ (or more correctly the image of $\Sigma$ ) be regarded as a subset of the Tychonoff compact space $X^{X}$, and define $E$ to be the closure of $\Sigma$ in this space. $E$ is a subgroup of the semigroup $X^{X}$ because $(\Sigma, X)$ is distal. (Ellis [6]; there $\Sigma$ is assumed to be a group, but as pointed out in [16] the arguments use only the semigroup property.) It follows

Received by the editors May 7, 1971 and, in revised form, March 20, 1972.

AMS (MOS) subject classifications (1970). Primary 43A60.

Key words and phrases. Weakly almost periodic function, fixed point theorem, Eberlein compact.

(1) Alfred P. Sloan Fellow. Research supported by NSF grant GP-18961. 
in particular that each $\sigma \in \Sigma$ is onto and therefore a homeomorphism. Letting $\Sigma^{*}$ be the subgroup of $E$ generated by $\Sigma$, we have that $\left(\Sigma^{*}, X\right)$ is a distal flow with Ellis group $E$. Below we give two consequences of these remarks.

1.1 Lemma. Let $(\Sigma, X)$ be a distal flow with $X$ compact Hausdorff. $X$ supports a regular Borel probability measure invariant under the transformations of $\Sigma$.

Proof. This follows from the existence of an invariant measure for $\left(\Sigma^{*}, X\right)$ ([8], [7]).

1.2 Lemma. Let $(\Sigma, X)$ and $\Sigma^{*}$ be as above, and let $\Sigma_{0}^{*} \subseteq \Sigma^{*}$ be the set of transformations of the form $\sigma^{-1}, \sigma \in \Sigma$. For each $x \in X, \Sigma x$ and $\Sigma_{0}^{*} x$ bave the same closure (namely Ex).

Proof. $\Sigma_{0}^{*}$ is a subsemigroup of $\Sigma^{*}$ which generates $\Sigma^{*}$ and therefore $E\left(\Sigma_{0}^{*}, X\right)=E\left(\Sigma^{*}, X\right)=E(\Sigma, X)$. The claim follows now because closure $\left(\Sigma_{0}^{*} x\right)=$ $E\left(\Sigma_{0}^{*}, X\right) x=E(\Sigma, X) x=$ closure $(\Sigma x)$.

Finally, recall that a flow $(\Sigma, X)$ is minimal if $\Sigma x$ is a dense subset of $X$ for every $x \in X$.

2. Flows on bitopological spaces. One often encounters a semigroup acting on a space which has two "natural" topologies. Our results are based on simple comparisons of dynamical properties of the flows in the competing topologies. We remark that our application of the Baire category theorem to obtain the set $C$ below with certain continuity properties is not new (we were unaware of this at the time [19] was written). Compare with [15]. So far as we know, the results themselves are new.

2.1 Lemma. Let $X$ be a space with topologies $\tau_{1}$ and $\tau_{2}$ such that

(a) $\left(X, \tau_{1}\right)$ is compact Hausdorff.

(b) $\left(X, \tau_{2}\right)$ is metrizable with compatible metric $D(\cdot, \cdot)$.

(c) For each $x \in X, D(x, \cdot)$ is $\tau_{1}$ continuous at all points of a $\tau_{1}$ residual set.

(d) $X$ bas a countable $\tau_{2}$ dense subset.

Let $(\Sigma, X)$ be a flow on the discrete space $X$, and assume

(i) $\left(\Sigma, X, \tau_{1}\right)$ is a minimal flow.

(ii) $(\Sigma, X, D)$ is a distal flow.

Then $\left(\Sigma, X, \tau_{1}\right)$ is a distal flow.

Proof. Let $x_{1}, x_{2}, \cdots$ be $\tau_{2}$ dense, and define, for each $n, \phi_{n}(x)=D\left(x, x_{n}\right)$. If $C_{n}$ is the $\tau_{1}$ residual set of points of $\tau_{1}$ continuity for $\phi_{n}$, define $C=\bigcap_{n=1}^{\infty} C_{n}$. $C \neq \varnothing$ by the Baire category theorem. We will show $D(x, \cdot)$ is $\tau_{1}$ continuous at $x$ for every $x \in C$. To this end fix $\epsilon>0$ and let $n$ be such that $D\left(x, x_{n}\right)<\epsilon / 3$. 
Since $x \in C$, there exists a $\tau_{1}$ neighborhood $W$ of $x$ such that $\left|\phi_{n}(w)-\phi_{n}(x)\right|<$ $\epsilon / 3, w \in W$. By the triangle inequality $D(x, w)<\epsilon, w \in W$. Now suppose $x, x^{\prime}$ are $\tau_{1}$ proximal. This means there exists a net $\left\{\sigma_{\nu}\right\}$ in $\Sigma$ and $y \in X$ such that $\tau_{1}-\lim _{\nu} \sigma_{\nu} x=y=\tau_{1}-\lim _{\nu} \sigma_{\nu} x^{\prime}$. Since $\left(\Sigma, X, \tau_{1}\right)$ is minimal, we may suppose $y \in C$. Therefore, $\lim _{\nu} D\left(\sigma_{\nu} x, \sigma_{\nu} x^{\prime}\right)=0$, which by the assumed $D$-distality implies $x=x^{\prime}$. The lemma is proved.

2.2 Proposition. Let the bypotheses of Lemma 2.1 bold with (d) and (i) replaced by

(d') The $\tau_{1}$ closure of every countable subset of $X$ bas a countable $\tau_{2}$ dense subset.

(i') $\left(\Sigma, X, \tau_{1}\right)$ is a flow (we do not assume minimality).

Then $\left(X, \tau_{1}\right)$ supports a regular Borel invariant probability measure.

Proof. Let $\Sigma_{0} \subseteq \Sigma$ be a countable subsemigroup, and let $X_{0} \subseteq X$ be a $\tau_{1}$ closed set such that $\left(\Sigma_{0}, X_{0}, \tau_{1}\right)$ is minimal. (The existence of $X_{0}$ is a wellknown consequence of Zorn's lemma.) $X_{0}$ has a countable $\tau_{1}$ dense subset, namely $\Sigma_{0} x$ for any $x \in X_{0}$, and therefore (d) is true for $\left(X_{0}, \tau_{2}\right)$. It follows by Lemma 2.1 that $\left(\Sigma_{0}, X_{0}, \tau_{1}\right)$ is distal, and therefore, by Lemma $1.1,\left(X_{0}, \tau_{1}\right)$ supports a $\Sigma_{0}$ invariant probability measure which we denote by $\mu\left(\Sigma_{0}\right)$. The collection $\left\{\mu\left(\Sigma_{0}\right) \mid \Sigma_{0} \subseteq \Sigma\right.$ a countable subsemigroup $\}$ is a net if the $\Sigma_{0}$ 's are ordered by inclusion, and if $\mu$ is the limit of a weak-* convergent subnet, $\mu$ is the desired measure. The proposition is proved.

2.3 Proposition. Let the bypotheses of Lemma 2.1 bold with (a) and (d) replaced by

$\left(\mathrm{a}^{\prime}\right)\left(X, \tau_{1}\right)$ is bomeomorphic to a weakly compact subset of Banacb space $B$. $\left(\left(X, \tau_{1}\right)\right.$ is an "Eberlein compact".)

(d') As in Proposition 2.2.

Then $\left(X, \tau_{1}\right)$ is metrizable, and $\left(\Sigma, X, \tau_{1}\right)$ is distal.

Proof. If $\left(X, \tau_{1}\right)$ is metrizable, then $X$ has a countable $\tau_{1}$ dense subset, and so, by ( $\left.\mathrm{d}^{\prime}\right), X$ has a countable $\tau_{2}$ dense subset. Distality is then a consequence of Lemma 2.1. We have left to prove metrizability. By Proposition 2.1, $\left(X, \tau_{1}\right)$ supports an invariant Borel probability measure $\mu$, which, by minimality, must assign positive measure to every nonempty $\tau_{1}$ open set. Thus, $\left(X, \tau_{1}\right)$ satisfies the "countable chain condition", meaning $X$ does not contain an uncountable collection of pairwise disjoint, nonempty, $\tau_{1}$ open sets. Corollary 4.6 of [17] asserts that a weakly compact subset of a Banach space is weakly metrizable if it satisfies the countable chain condition, and so we are done. (The existence of $\mu$ makes the full strength of Rosenthal's theorem unnecessary. For the unit ball of $L^{2}(\mu)$ is a weakly compact weak- ${ }^{*}$ total set $L$ of the dual of 
$C\left(X, \tau_{1}\right)$ and by Proposition 1 of $[1] C\left(X, \tau_{1}\right)$ contains a weakly compact total set $K$ which in particular is point separating on $X$. By the Dunford-Pettis property [11] $K$, regarded as a subset of $C(L)$, is norm compact, hence norm separable, and therefore $K$ is weakly separable (hence norm separable in $C(X)$ ), and this implies $\left(X, \tau_{1}\right)$ is metrizable. Rosenthal proves in [13, Theorem 4.5] that a compact space $X$ satisfies the countable chain condition if and only if every weakly compact subset of $C(X)$ is norm separable.)

2.4 Theorem. Let $B$ be a conjugate Banach space equipped with a weak-* topology $\tau_{1}$, and let $\tau_{2}$ be the norm topology. Let $D(x, y)=\|x-y\|, x, y \in B$, where $\|\cdot\|$ is the norm on $B$. Suppose $\Sigma$ is a semigroup of bounded $\tau_{1}$ continuous linear operators on $B$, and let $X \subseteq B$ be a $\tau_{1}$ compact, $\Sigma$-invariant set. If the assumptions ( $\mathrm{d}^{\prime}$ ) and (ii) of Proposition 2.2 are valid for $\Sigma, X, \tau_{1}, \tau_{2}$, and $D$, the weak-* closed convex bull of $X$ contains a fixed point for $\Sigma$.

Proof., Conditions (a), (b), (d'), ( $\left.\mathrm{i}^{\prime}\right)$, and (ii) are automatically satisfied. For each $x \in X, D(x, \cdot)$ is $\tau_{1}$ lower semicontinuous and therefore continuous at the points of a $\tau_{1}$ residual subset of $X$. It follows from Proposition 2.2 that $\left(X, \tau_{1}\right)$ supports a $\Sigma$-invariant probability measure $\mu$, and the desired fixed point is $x_{0}=$ ${ }_{X}^{\int x \mu}(d x)$, the integral being taken in the weak-* sense.

2.5 Corollary. Let $X$ be a weakly compact subset of a Banacb space $B$, and let $\Sigma$ be a semigroup of bounded linear operators on $B$ which leaves $X$ invariant. If $\Sigma$ is norm distal on $X$, the weakly closed ( = norm closed) convex bull of $X$ contains a fixed point for $\Sigma$.

Proof. Condition $\left(d^{\prime}\right)$ is automatic here because, by the Banach-Mazur theorem, the weak closure of a countable set is norm separable. Theorem 2.4 (or Proposition 2.2 of course) applies directly because we can regard $X, B$ as subsets of the bidual $B^{* *}$, where the weak- ${ }^{*}$ topology on $X$ is simply the original weak topology.

Remark. If $X$ is also convex, Corollary 2.5 is just the Ryll-Nardzewski fixed point theorem (for Banach spaces). Our fixed point theorem stands to that of RyllNardzewski as Namioka's fixed point theorem [16, Theorem 4.3] stands to Hahn's fixed point theorem [12].

2.6 Lemma. Let the assumptions of Lemma 2.1 be strengthened by

(ii') $(\Sigma, X, D)$ is equicontinuous and distal. Then $\tau_{1}=\tau_{2}$, and in particular $\left(\Sigma, X, \tau_{1}\right)$ is equicontinuous.

Proof. Let $C$ be the set constructed in Lemma 2.1. Given $\epsilon>0$, choose $\delta>0$ so that if $D\left(x, x^{\prime}\right)<\delta$, then $D\left(\sigma x, \sigma x^{\prime}\right)<\epsilon$ for all $\sigma \in \Sigma$. Choose $y \in C$, and let $W$ be a $\tau_{1}$ neighborhood of $y$ on which $D(y, w)<\delta / 2$ (see the proof of Lemma 2.1). If $w, w^{\prime} \in W$, then $D\left(w, w^{\prime}\right)<\delta$ by the triangle inequality. By 
Lemma 2.1 and the discussion preceding Lemma 1.1 each $\sigma \in \Sigma$ is one-one onto. Let $\Sigma_{0}^{*}=\left\{\sigma^{-1} \mid \sigma \in \Sigma\right\}$. If $x \in X$, the fact that $\Sigma x$ is $\tau_{1}$ dense implies, by Lemmas 2.1 and 1.2 , that $\Sigma_{0}^{*}$ is $\tau_{1}$ dense. In particular, there exists $\lambda \in \Sigma_{0}^{*}$ with $\lambda x \in W$. The definition of $\Sigma_{0}^{*}$ therefore implies $\{\sigma W \mid \sigma \in \Sigma\}$ is an open cover of $X$, and so there exist $\sigma_{1}, \cdots, \sigma_{t} \in \Sigma$ such that $X=\sigma_{1} W \cup \cdots \cup \sigma_{t} W$. The choice of $\delta$ and $W$ implies $\sigma_{1} w, \cdots, \sigma_{t} w$ is an $\epsilon$-net for any $w \in W$. Thus $(X, D)$ is totally bounded. We will prove $(X, D)$ is complete and that the identity map is continuous from $(X, D)$ to $\left(X, \tau_{1}\right)$. Both statements are a consequence of the following: Let $\left\{y_{n}\right\}$ be a $D$-Cauchy sequence, and let $z$ be a $\tau_{1}$ cluster point of $\left\{y_{n}\right\}$. Then $\lim _{n \rightarrow \infty} D\left(y_{n}, z\right)=0$. To see this suppose $\epsilon, \delta$, and $W$ are as above, and let $\lambda=$ $\sigma^{-1} \in \Sigma_{0}^{*}$ be such that $\lambda z \in W$. Infinitely often we have $\lambda y_{n} \in W$, and for each such $D\left(y_{n}, z\right)=D\left(\sigma \lambda y_{n}, \sigma \lambda z\right)<\epsilon$. It follows there is a subsequence of $\left\{y_{n}\right\}$ which is $D$-convergent to $z$, and since $\left\{y_{n}\right\}$ is Cauchy, $D\left(y_{n}, z\right) \rightarrow 0$. The lemma is proved.

2.7 Theorem. Let $X$ be a weakly compact subset of a Banach space $B$, and let $\Sigma$ be a semigroup of bounded linear operators on $B$ leaving $X$ invariant. Assume

(A) $(\Sigma, X)$ is minimal in the weak topology.

(B) $(\Sigma, X)$ is distal and equicontinuous in the norm topology.

Then $X$ is norm compact.

Proof. By Proposition 2.3, $X$ is weakly metrizable and therefore weak and norm separable. Apply Lemma 2.6.

Remark. If $\Sigma$ is a group, the distality assumptions made in 2.6 and 2.7 are unnecessary. In particular, Lemma 2.6 , which plays a central role in $\$ 3$, does not need Lemma 2.1 in the group case.

3. The algebra $K(\Sigma)$. In this section $\Sigma$ is assumed to be a group. We use $l^{\infty}=l^{\infty}(\Sigma)$ to denote the Banach space of bounded, complex-valued functions $\Sigma$. To each $f \in l^{\infty}$ we associate a compact space $X$, the weak-* closure of the set of right translates of $f$. ( $l^{\infty}$ is the dual of the space of absolutely summable functions on $\Sigma$, and "weak-", is with respect to this duality.) Letting $\Sigma$ act by right translation on $X_{f}$, we obtain a flow $\left(\Sigma, X_{f}\right)$.

If $\Sigma_{1}, \Sigma_{2}$ are subsets of $\Sigma$, and if $f \in l^{\infty}$, we shall denote by $X\left(f, \Sigma_{1}, \Sigma_{2}\right) \subseteq$ $l^{\infty}\left(\Sigma_{2}\right)$ the set of restrictions to $\Sigma_{2}$ of the weak- ${ }^{*}$ closure (in $l^{\infty}(\Sigma)$ ) of the right translates of $f$ by elements of $\Sigma_{1}$. Thus, $X(f, \Sigma, \Sigma)=X_{f}$.

3.1 Definition. $K(\Sigma)$ is the set of $f \in l^{\infty}$ having the property that $X\left(f, \Sigma_{1}, \Sigma_{2}\right)$ is norm separable for all countable sets $\Sigma_{1}, \Sigma_{2} \subseteq \Sigma$.

3.2 Example. If $X$ is weakly compact in $l^{\infty}(f$ is "right weakly almost periodic"), then $X\left(f, \Sigma_{1}, \Sigma\right)$ is norm separable whenever $\Sigma_{1}$ is countable by the 
Banach-Mazur theorem, and therefore $f \in K(\Sigma)$. The function $f$ on the integers ( = $\mathbf{Z}$ ) defined to be 0 for $n \leq 0$ and one for $n>0$ belongs to $K(\mathbf{Z})$ but is not weakly almost periodic.

3.3 Lemma. $K(\Sigma)$ is a uniformly closed, conjugation closed, right and left translation invariant subalgebra of $l^{\infty}$.

Proof. We omit the routine details.

3.4 Lemma. If $f \in K(\Sigma)$, then $X, \subseteq K(\Sigma)$.

Proof. Fix $g \in X_{f}$ and $\Sigma_{1}, \Sigma_{2} \subseteq \Sigma$ countable. To say $g \in X_{f}$ is to say there exists a net $a=\left\{\alpha_{\nu}\right\}$ in $\Sigma$ such that $g(\sigma)=\lim _{\nu} f\left(\sigma \alpha_{\nu}\right)$ for all $\sigma$. Let $\Sigma^{(1)}$ be the (countable) group generated by $\Sigma_{1}, \Sigma_{2}$. There exists a sequence $\alpha^{(1)}=\left\{a_{n 1}\right\}$ in $\Sigma$ such that $g(\sigma)=\lim _{n} f\left(\sigma \alpha_{n 1}\right), \sigma \in \Sigma^{(1)}$. Let $\Sigma^{(2)}$ be the (countable) group generated by $\Sigma^{(1)}$ and $\alpha^{(1)}$. Then choose $\alpha^{(2)}$ for $\Sigma^{(2)}$ with properties analogous to those of $a^{(1)}$. Proceeding in this fashion, we obtain an increasing sequence $\Sigma^{(k)}$ of countable subgroups of $\Sigma$, and a sequence $\alpha^{(k)}$ of sequences from $\Sigma$, such that (i) $\lim _{n} f\left(\sigma \alpha_{n k}\right)=g(\sigma), \sigma \in \Sigma^{(k)}$ and (ii) $\Sigma^{(k)} \cup \alpha^{(k)} \subseteq \Sigma^{(k+1)}$. A diagonal argument shows there is a sequence $\alpha$ in $\Sigma_{0}=\bigcup_{k} \Sigma^{(k)}$ such that $g(\sigma)=$ $\lim _{n} f\left(\sigma \alpha_{n}\right), \sigma \in \Sigma_{0}$. Since $\Sigma_{0}$ is a group, $X\left(g, \Sigma_{0}, \Sigma_{0}\right) \subseteq X\left(f, \Sigma_{0}, \Sigma_{0}\right)$. Thus, $X\left(g, \Sigma_{1}, \Sigma_{2}\right)$ is norm separable because $X\left(f, \Sigma_{0}, \Sigma_{0}\right)$ is norm separable. The lemma is proved.

According to [14] there exists a net $a=\left\{a_{\nu}\right\}$ in $\Sigma$ with the properties

(a) $S_{u} f(\sigma)=\lim _{\nu} f\left(\sigma \alpha_{\nu}\right)$ exists for all $f$ and $\sigma$.

(b) $s_{u}^{2} f=S_{u} f$, all $f$.

(c) $S_{u} f$ is right minimal.

We recall that a function $g$ is right minimal if and only if $\left(\Sigma, X_{g}\right)$ is a minimal flow. $S_{u}$ is called a (right) minimal idempotent. Every $f \in l^{\infty}$ decomposes as $f=g+b, g=S_{u} f, b=f-g$, in which $g$ is right minimal and $S_{u} b=0$. If $f \in K(\Sigma)$, then because $S_{u} f \in X$, both $g$ and $b$ are $K(\Sigma)$. Shortly we will see that $g$ is almost periodic $\left(X_{g}\right.$ is norm compact).

3.5 Proposition. If $\Sigma$ is a countable group, then any right minimal element of $K(\Sigma)$ is almost periodic.

Proof. Suppose $f \in K(\Sigma)$ is right minimal. Then Lemma 2.6 applies to $\left(\Sigma, X_{f}\right)$ with $\tau_{1}=$ weak $^{*}$ topology, $\tau_{2}=$ norm topology, $D=$ norm metric. $\left(\Sigma, X_{f}, D\right)$ is distal and equicontinuous because $\Sigma$ acts by norm isometries. By Lemma 2.6, $X_{f}$ is norm compact, and so $f$ is almost periodic.

3.6 Lemma. If $f \in l^{\infty}$ is right minimal, and if $\Sigma_{1} \subseteq \Sigma$ is a countable set, there is a countable subgroup $\Sigma_{2}, \Sigma_{1} \subseteq \Sigma_{2} \subseteq \Sigma$, sucb that $f$ restricted to $\Sigma_{2}$ is right minimal. 
Proof. A bounded function $g$ on a group $\Gamma$ is right minimal if and only if for every $\epsilon>0$ and finite set $F \subseteq \Gamma$ there exist $\gamma_{1}, \ldots, \gamma_{p} \in \Gamma$ such that $\gamma_{1} C \cup \ldots$ $\cup \gamma_{p} C=\Gamma$, where

$$
C=C_{\epsilon}(F)=\left\{\tau\left|\max _{\sigma \epsilon F}\right| f(\sigma \tau)-f(\sigma) \mid<\epsilon\right\}
$$

(see [14]). Fixing $\Sigma_{1} \subseteq \Sigma$ countable, we associate to every finite set $F \subseteq \Sigma_{1}$, cardinality $(F)=n>0$, elements (not uniquely determined) $\sigma_{1}, \ldots, \sigma_{p}, p=p(F)$ such that $\sigma_{1} C_{\epsilon}(F) \cup \ldots \cup \sigma_{p} C_{\epsilon}(F)=\Sigma, \epsilon=1 / n$. Then define $\bigwedge(F)$ to be the smallest group containing $F$ and $\sigma_{1}, \ldots, \sigma_{p}$. Let $q\left(\Sigma_{1}\right)=\bigcup \wedge(F)$, the union being over all finițe sets $F \subseteq \Sigma_{1}$. Inductively, we define $\Sigma^{(1)}=q\left(\Sigma_{1}\right), \Sigma^{(n+1)}=$ $q\left(\Sigma^{(n)}\right), n \geq 1$. Define $\Sigma_{2}=\bigcup_{n=1}^{\infty} \Sigma^{(n)} . \Sigma_{2}$ is a group, and we claim $f$ is right minimal on $\Sigma_{2}$. To see this fix $\epsilon>0$ and $F_{0} \subseteq \Sigma_{2}$ finite, and choose $F \supseteq F_{0}$, also finite, so that $1 / n<\epsilon, n=$ cardinality $(F)$. Since $F \subseteq \Sigma^{(K)}$ for some $K$, there exist $\sigma_{1}, \ldots, \sigma_{p} \in \Sigma_{2}$ (actually in $\Sigma^{(K+1)}$ ) such that $\sigma_{1} C_{\epsilon}(F) \cup \cdots \cup \sigma_{p} C_{\epsilon}(F)=$ $\Sigma$ (since $\epsilon>1 / n)$. Since $\sigma_{1}, \ldots, \sigma_{p} \in \Sigma_{2}, \sigma_{1} C_{\epsilon}^{\prime}(F) \cup \ldots \cup \sigma_{p} C_{\epsilon}^{\prime}(F)=\Sigma_{2}$, where $C_{\epsilon}^{\prime}(F)=C_{\epsilon}(F) \cap \Sigma_{2}$. That $f$ is minimal on $\Sigma_{2}$ now follows from the "if" of the result quoted above.

If $f \in K(\Sigma)$, then clearly $f \in K\left(\Sigma^{\prime}\right)$ for any subgroup $\Sigma^{\prime}$ of $\Sigma$, if we view $f$ as a function on $\Sigma^{\prime}$. If $f$ is right minimal on $\Sigma$, and if $f \in K(\Sigma)$, then for every countable set $\Sigma_{1}$ there exists a countable subgroup $\Sigma_{2} \supseteq \Sigma_{1}$ such that (a) $f \in K\left(\Sigma_{2}\right)$, and (b) $f$ is right minimal on $\Sigma_{2}$. Thus, $f$ is almost periodic restricted to this same $\Sigma_{2}$. If $\Sigma_{1}$ is any countable subgroup, then because almost periodicity is an hereditary property, $f$ is almost periodic on $\Sigma_{1}$. That is, if $f \in K(\Sigma)$ is right minimal, it is almost periodic when restricted to any countable subgroup. With this observation we prove

3.7 Theorem. If $f \in K(\Sigma)$ is right minimal, $f$ is almost periodic.

Proof. A necessary and sufficient condition that $f$ fail to be almost periodic is that there exist an $\epsilon>0$ and a sequence $\sigma_{1}, \sigma_{2}, \ldots$ in $\Sigma$ such that for all $i \neq j$

$$
\sup \left|f\left(\sigma \sigma_{i}\right)-f\left(\sigma \sigma_{j}\right)\right| \geq \epsilon .
$$

A necessary and sufficient condition for $(3.8)$ to hold is that there exist a countable subgroup $\Sigma_{1}$ of $\Sigma$, containing $\sigma_{1}, \sigma_{2}, \ldots$, such that (3.8) holds on $\Sigma_{1}$. Since $f$ is almost periodic on $\Sigma_{1}$, (3.8) cannot hold on $\Sigma_{1}$, and therefore it cannot hold in $\Sigma$. Thus $f$ is almost periodic.

Returning to the decomposition preceding Proposition 3.5 we see that if $f=$ $g+b, g=S_{u} f, b=f-g$, for $f \in K(\Sigma)$, then $g$ is almost periodic. Using this and basic facts about weakly almost periodic functions, we shall prove 
3.9 Theorem (Eberlein, de Leeuw and Glicksberg, Ryll-Nardzewski). If $f$ is weakly almost periodic, then $f=g+b$, where $g$ is almost periodic and $b$ has the property that the constant function 0 is in the norm closed convex bull of both the right and left translates of its absolute value. These properties uniquely determine $g$ and $h$.

Proof. Let $S_{u}$ be a right minimal idempotent, and set $g=S_{u} f, b=f-g \cdot g$ is almost periodic by 3.7. As for $h$, it is weakly almost periodic, and therefore $|b|$ is weakly almost periodic [4]. Since $S_{u}|b|=\left|S_{u} b\right|=0$, and since $S_{u}|b|$ is a pointwise limit of right translates of $|b|, 0$ is a weak limit of right translates of $|b|$. By the Banach-Mazur theorem $|b|$ has 0 in the norm closed convex hull of its right translates. Moreover, any function which is a (weak- $\left.{ }^{*}\right)$ limit of left translates of $|b|$ has the same property. Now $|b|$ is also weakly almost periodic on the left [10]. If $T_{v}$ is a left minimal idempotent, then by the left-hand version of Theorem 3.7, $T_{v}|b|$ is almost periodic, and by the remark just made, $T_{v}|b|$ has 0 in the norm closed convex hull of its right translates. Thus, $T_{v}|b|=0=T_{v} b$, and so $|b|$ has 0 in the norm closed convex hull of its left translates. If $f=g^{\prime}+b^{\prime}$ with similar properties, then $T_{v}\left|b^{\prime}\right|=0=T_{v} b^{\prime}$ by the above, and so $g=T_{v} f=g^{\prime}$. (If $T_{v}$ is a minimal idempotent, $T_{v} g=g$ for any almost periodic $g$, as is easily proven.) The theorem is proved.

It seems not to have been noted before that Godement's decomposition theorem for positive definite functions can be derived from 3.9. We indicate this here. The referee has pointed out to us that Godement's theorem also follows easily from the (post Ryll-Nardzewski) the ory of weakly almost periodic representations.

3.10 Lemma. Let $S_{u}$ be a right minimal idempotent, and let $\gamma \in \Sigma$. Then $S_{\gamma-1} S_{u} S_{\gamma}$ is a right minimal idempotent, where if $\alpha=\left\{\alpha_{\nu}\right\}$ defines $S_{u}$,

$$
S_{\gamma-1} S_{u} S_{\gamma} f(\sigma)=\lim _{\nu} f\left(\sigma \gamma^{-1} \alpha_{\nu} \gamma\right) \text {. }
$$

Proof. Idempotency is an easy consequence of (3.11). Right minimality follows because $S_{u} f^{\gamma}$ is right minimal, where $f^{\gamma}(\sigma)=f(\sigma \gamma)$, the right minimal functions are closed under translations, and $(3.11)$ is just $\left(S_{u} f^{\gamma}\right)^{\gamma-1}$.

3.12 Lemma. Let $S_{u}$ and $a$ be as above, and let $f$ be weakly almost periodic. Then for all $\sigma \in \Sigma$

$$
\begin{aligned}
S_{u} f(\sigma) & =\lim _{\nu} f\left(\sigma \alpha_{\nu}\right) \\
& =\lim _{\nu} f\left(\sigma \alpha_{\nu}^{-1}\right) \\
& =\lim _{\nu} f\left(\alpha_{\nu}^{-1} \sigma\right) \\
& =\lim _{\nu} f\left(\alpha_{\nu} \sigma\right) .
\end{aligned}
$$


Proof. Recall from earlier discussion that $S_{v} f=S_{u} f=T_{v} f=T_{u} f$ for all left and right minimal idempotents $S_{v}, S_{u}, T_{v}, T_{u}$. The involution $x \rightarrow x^{-1}$ interchanges right and left minimal idempotents, and therefore the first and third lines of (3.13) are equal. That the first and fourth lines are equal is a consequence of (3.10) and the fact that the fourth line, for fixed $\sigma$, is just $\left(S_{\sigma-1} S_{u} S_{\sigma}\right) f(\sigma)$. Equality of lines two and three is for similar reasons. The lemma is proved.

Let $H$ be a Hilbert space, and suppose $\sigma \rightarrow U_{\sigma}$ is a unitary representation of $\Sigma$ on $H$. According to Eberlein [4], if $x, y \in H$, the function $f_{x y}$, defined by $f_{x y}(\sigma)=\left\langle x, U_{\sigma} y\right\rangle(\langle\rangle=$, inner product on $H)$ is weakly almost periodic. Therefore, if we define $P$ on $H$ weakly by $\langle x, P y\rangle=\lim _{\nu}\left\langle x, U_{a_{\nu}} y\right\rangle$, Lemma 3.12 implies $P U_{\sigma}=U_{\sigma} P$ for all $\sigma$ and $P=P^{*}$. Now Godement's representation of bounded positive definite functions on $\Sigma$ tells us a function $g$ is bounded positive definite if and only if there exists $H, U_{\sigma}$, and $x \in H$ with $g=f_{x x^{*}}$. We obtain from all this that, for $g=f_{x x}$ positive definite,

$$
\begin{aligned}
g(\sigma) & =S_{u} f(\sigma)+f(\sigma)-S_{u} f(\sigma) \\
& =\left\langle P x, U_{\sigma} P x\right\rangle+\left\langle(I-P) x, U_{\sigma}(I-P) x\right\rangle=f_{P x P x}+f_{(I-P) x(I-P) x}
\end{aligned}
$$

is a sum of positive definite functions, one almost periodic, and one with properties similar to that of $b$ in the Eberlein et al. theorem. As mentioned earlier, this decomposition is due to Godement.

The results of the present section have appeared in our unpublished notes [19].

4. Remarks. Since this paper was written, Professor I. Namioka has informed us that the approach of $\$ 2$ yields the following generalization of the Ryll-Nardzewski fixed point theorem for locally convex spaces. We omit the details.

Theorem (Namioka). Let $(E, \mathcal{J})$ be a locally convex Hausdorff linear topological space, let $C$ be a nonvoid weakly compact convex subset of $E$, and let $\Sigma$ be a semigroup of weakly continuous affine maps of $C$ into itself. If $\Sigma$ is $\mathfrak{T}$ noncontracting on a $\Sigma$ stable weakly closed nonempty subset $K$ of $C$, then there is a common fixed point of $\Sigma$ in the closed convex bull of $K$.

Let $(\Sigma, \mathcal{T})$ be a topological group, and let $C(\Sigma, \mathscr{T})$ be the Banach space of bounded $\mathcal{T}$ continuous functions on $\Sigma$. Recall that $f \in C(\Sigma, \mathfrak{T})$ is weakly almost periodic on $(\Sigma, \mathcal{J})$ if the set of right translates of $f$ is weakly relatively compact in $C(\Sigma, \mathfrak{T})$. Since the inclusion $C(\Sigma, \mathfrak{T}) \rightarrow l^{\infty}(\Sigma)$ is a weak isomorphism into, it is equivalent to say that $f$ is weakly almost periodic in the sense of $\$ 3$. Taking into account the fact that $g$ and $b$ in 3.9 lie in the closed translation invariant subspace generated by $f$, we see that both $g$ and $b$ are continuous. Therefore 3.9 (and its proof) imply the decomposition theorem in full generality. 


\section{REFERENCES}

1. D. Amir and J. Lindenstrauss, The structure of weakly compact sets in Banach spaces, Ann. of Math. (2) 88 (1968), 35-46. MR 37 \#4562.

2. K. de Leeuw and I. Glicksberg, Applications of almost periodic compactifications, Acta Math. 105 (1961), 63-97. MR 24 \#A1632.

3. N. Dunford and J. T. Schwartz, Linear operators. I: General theory, Pure and Appl. Math., vol. 7, Interscience, New York, 1958. MR 22 \#8302.

4. W. F. Eberlein, Abstract ergodic theorems and weakly almost periodic functions, Trans. Amer. Math. Soc. 67 (1949), 217-240. MR 12, 112.

5. R. Ellis, A semigroup associated with a transformation group, Trans. Amer. Math. Soc. 94 (1960), 272-281. MR 33 \#A961.

6. - Distal transformation groups, Pacific J.Math. 8 (1958), 401-405. MR 21 \#96.

7. - Lectures on topological dynamics, Benjamin, New York, 1969. MR 42 \#2463.

8. H. Furstenberg, The structure of distal flows, Amer. J. Math. 85 (1963), 477-515. MR $28 \# 602$.

9. R. Godement, Les fonctions de type positif et la théorie des groupes, Trans. Amer. Math. Soc. 63 (1948), 1-84. MR 9, 327.

10. A. Grothendieck, Critères de compacité dans les espaces fonctionnels généraux, Amer. J. Math. 74 (1952), 168-186. MR 13, 857.

11. - Sur les applications linéaires faiblement compactes d'espaces du type $C(K)$, Canad. J. Math. 5 (1953), 129-173. MR 15, 438.

12. F. Hahn, A fixed-point theorem, Math. Systems Theory 1 (1967), 55-57. MR 34 $\# 8202$.

13. J. L. Kelley, General topology, Van Nostrand, Princeton, N. J., 1955. MR 16, 1136.

14. A. Knapp, Decomposition theorem for bounded uniformly continuous functions on a group, Amer. J. Math. 88 (1966), 902-914. MR 34 \#8212.

15. I. Namioka, Neighborhoods of extreme points, Israel J. Math. 5 (1967), 145-152. MR $36 \# 4323$.

16. - Right topological groups, distal flows, and a fixed point theorem (preprint).

17. H. P. Rosenthal, $O_{n}$ injective Banach spaces and the spaces $L^{\infty}(\mu)$ for finite measures $\mu$, Acta Math. 124 (1970), 205-248. MR $41 \# 2370$.

18. C. Ryll-Nardzewski, On fixed points of semigroups of endomorphisms of linear spaces, Proc. Fifth Berkeley Sympos. Math. Statist. and Probability (Berkeley, Calif., 1965/66), vol. II: Contributions to Probability Theory, part 1, Univ. of California Press, Berkeley, Calif., 1967, pp. 55-61. MR 35 \#5977.

19. W. Veech, Generalizations of almost periodic functions, Mimeographed Notes, 58 pp.

SCHOOL OF MATHEMATICS, INSTITUTE FOR ADVANCED STUDY, PRINCETON, NEW JERSEY 08540

Current address: Department of Mathematics, Rice University, Houston, Texas 77001 